\title{
A Clinical Decision Support Platform for the Risk Stratification, Diagnosis, and Prediction of Coronary Artery Disease Evolution
}

\author{
Antonis Sakellarios, Panagiotis Siogkas, Eleni Georga, Nikolaos Tachos, Vassiliki Kigka, Panagiota \\ Tsompou, Ioannis Andrikos, Georgia S. Karanasiou, Silvia Rocchiccioli, Joao Correia, Gualtriero \\ Pelosi, Paolo Stofella, Nenad Filipovic, Oberdan Parodi, Dimitrios I. Fotiadis, Member, IEEE
}

\begin{abstract}
SMARTool aims to the accurate risk stratification of coronary artery disease patients as well as to the early diagnosis and prediction of disease progression. This is achieved by the acquisition of data from about 300 patients including computed tomography angiographic images, clinical, molecular, biohumoral, exposome, inflammatory and omics data. Data are collected in two time points with a follow-up period of approximately 5 years. In the first step, data mining techniques are implemented for the estimation of risk stratification. In the next step, patients, who are classified as medium to high risk are considered for coronary imaging and computational modelling of blood flow, plaque growth and stenosis severity assessment. Additionally, patients with increased stenosis are selected for stent deployment. All the above modules are integrated in a cloud-based platform for the clinical decision support (CDSS) of patients with coronary artery disease. The work presents preliminary results employing the SMARTool dataset as well as the concept and architecture of the under development platform.
\end{abstract}

\section{INTRODUCTION}

Cardiovascular disease (CVD) has been established as one of the leading causes of death in western societies having social, economic and medical impact [1]. Atherosclerosis is the main form of CVD presenting a local phenotype affected however by systemic factors. More specifically, systemic factors and comorbid conditions such as hypertension and diabetes may accelerate the atherosclerotic process. Additionally, atherosclerosis has local manifestations affected by the hemodynamics and in particular the low endothelial shear stress (ESS). Low ESS is found to have a multifactorial effect to arterial physiology, for instance to gene expression, to endothelial permeability and to

*This work is partially funded by the European Commission: Project SMARTOOL, "Simulation Modeling of coronary ARTery disease: a tool for clinical decision support - SMARTool" GA number: 689068).

P.K. Siogkas, P. Tsompou, V. Kigka, Eleni I. Georga, Ioannis Andrikos and D.I. Fotiadis are with the Unit of Medical Technology and Intelligent Information Systems, Department of Materials Science and Engineering, University of Ioannina, Ioannina, GR 45110 Greece.

N. Filipovic is with BioIRC, Prvoslava Stojanovica 6, Kragujevac, 34000, Serbia.

S. Rocchiccioli, G. Pelosi and O. Parodi are with Institute of Clinical Physiology, National Research Council, 56124, Pisa, Italy (email: pelosi@ifc.cnr.it; oberdan.parodi@ virgilio.it)

Joao Correia is with Biotronics3D, 5 Greenwich View Place, London E14 9NN, United Kingdom (email: jcorreia@ biotronics3d.com).

Paolo Stofella is with EXPRIVIA, Molfetta (Bari), Viale A. Olivetti 11, 70056, Italy (email: paolo.stofella@exprivia.it).

A.I Sakellarios, P.K. Siogkas, N.S Tachos, G.S. Karanasiou and D.I. Fotiadis are with the Dept. of Biomedical Research, FORTH-IMBB, GR 45110 Ioannina, Greece (corresponding author phone: +302651009006; fax: +302651008889; e-mail: fotiadis@cc.uoi.gr). inflammation. Several clinical factors contribute to the formation of atherosclerotic plaque including age, gender, hypertension, hyperlipidemia, and other. Since atherosclerosis is affected by several factors, the process is multifactorial and starts from the accumulation of low density lipoproteins (LDL) in the arterial wall and their oxidation. This process triggers an inflammatory response in the arterial wall causing the monocyte migration and their transformation into macrophages. Consequently, foam cells are formed and the smooth muscle cells proliferate causing the wall thickening.

The recent years computational modelling is used for the understanding of the mechanisms of atherosclerosis. The majority of the studies focus on the blood flow modelling and the relation of low ESS with plaque formation [2, 3]. Besides the hemodynamics studies, LDL transport modelling is performed and its accumulation is associated to plaque formation. Most of these studies assume that endothelial membrane is a semi-permeable biological membrane with a constant permeability. Finally, very recently proof-of-concept studies were presented simulating the major steps of the atherosclerotic plaque formation process. One major outcome of the last decade is the acquisition and collection of big data coming from several categories including imaging, molecular, clinical, omics, lipidomics and inflammatory markers. The aim of this collection is the identification of potential risk factors and biomarkers for the risk stratification and the early diagnosis and prognosis of the disease. However, the main limitation of most of these attempts is that there is no comprehensive study or clinical trial, which collects data from all the above categories in two time points to estimate the progression of atherosclerosis.

SMARTool project aims to the collection of data from approximately 300 patients in two time points including computed tomography coronary angiography (CTCA) imaging, clinical data, biohumoral, exposome, inflammatory, molecular, lipidomics and omics from mRNA. The collected data are analysed and processed using machine learning algorithms in order to provide risk stratification of coronary artery disease (CAD) existence. In case of medium to high risk, CTCA is utilized for the $3 \mathrm{D}$ reconstruction of coronary arteries, the computational modelling of plaque progression for prediction Decision Support System (DSS), the noninvasive fractional flow reserve (FFR) for diagnosis DSS and the stent deployment modeling for treatment DSS. The proposed approach is integrated into a novel one of its kind cloud-based platform. The current work presents the concept and architecture of the proposed platform as well as the preliminary results of the data analysis. 


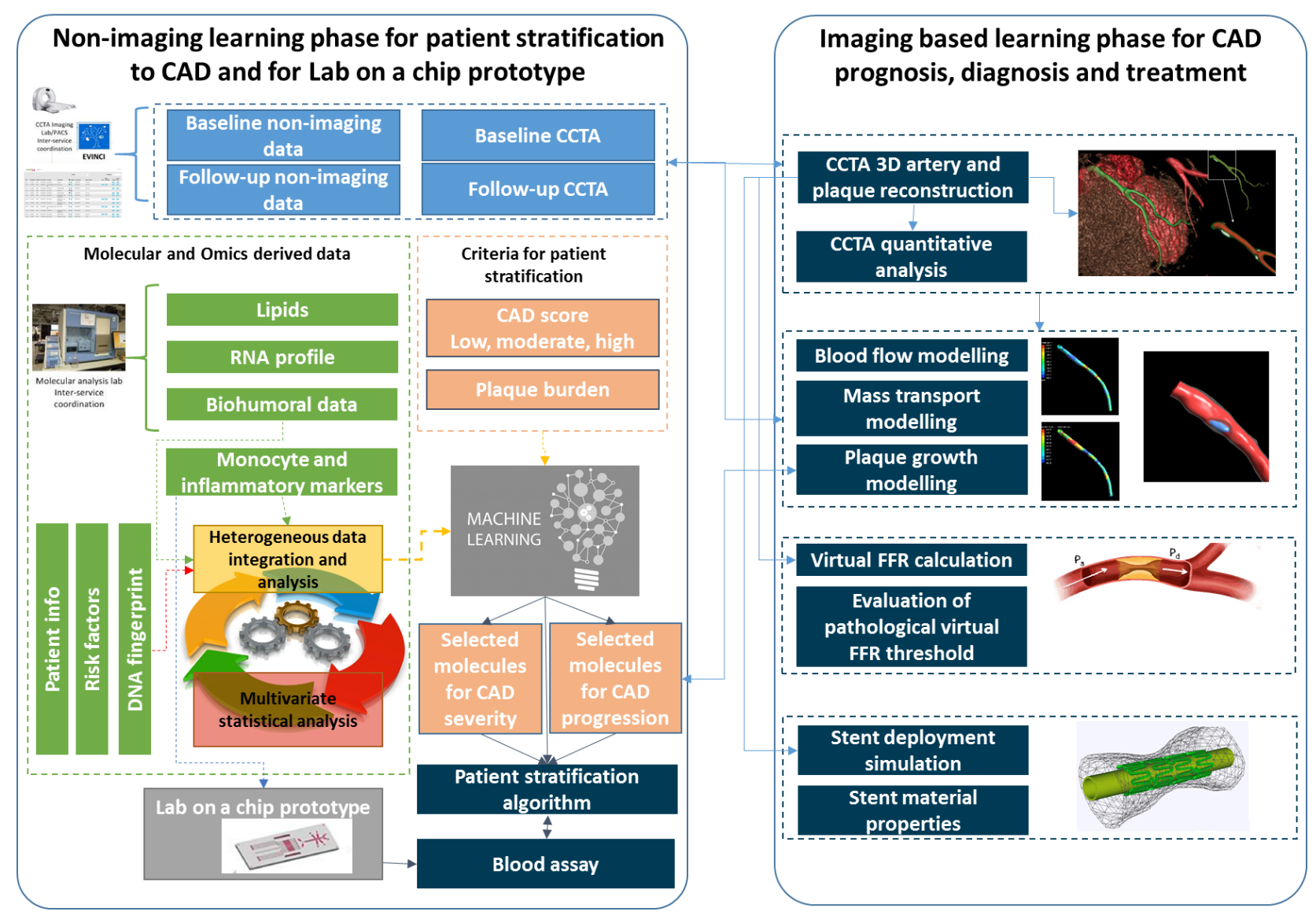

Figure 1. The overall concept of SMARTool platform.

\section{MATERIAL AND METHODS}

\section{A. Concept and approach}

The overall concept of SMARTool is presented in Figure 1. The collected data includes both imaging data (CTCA) and clinical, molecular and cellular data (clinical records, phenotype, genotype). As shown in Fig. 1, all non-imaging data of baseline and follow-up are analyzed using statistical approaches as well as using machine learning techniques. The aim is to identify biomarkers and independent predictors of CAD severity and progression. The identified markers are integrated into a CAD risk stratification algorithm.

The non-imaging phase of SMARTool is connected with an imaging based DSS. The proposed system consists of modules of DSS for diagnosis, prognosis and treatment. More specifically, the identified patients of medium-high risk from the risk stratification algorithm are called for a CTCA imaging, data of which are later used for the 3D reconstruction of the coronary arteries and the calculation of several geometric characteristics such as lumen area, plaque burden, calcified and non-calcified plaques, etc. The reconstructed geometries are utilized for blood flow and plaque growth modelling (prognosis DSS), non-invasive Flow Fractional Reserve (FFR) assessment (diagnosis DSS) and stent deployment (treatment DSS).

\section{B. Data acquisition}

Patient-specific data are collected from a retrospective database of EVINCI study [4], and it is considered as baseline information, while 262 patients are re-enrolled after $5 \pm 2$ years for a follow-up re-evaluation. A clinical case report (CRFA) platform is developed for data storing and simple analytics as depicted in Fig. 2. The platform ensures through internal controls, that no faulty entries or missing values are added.

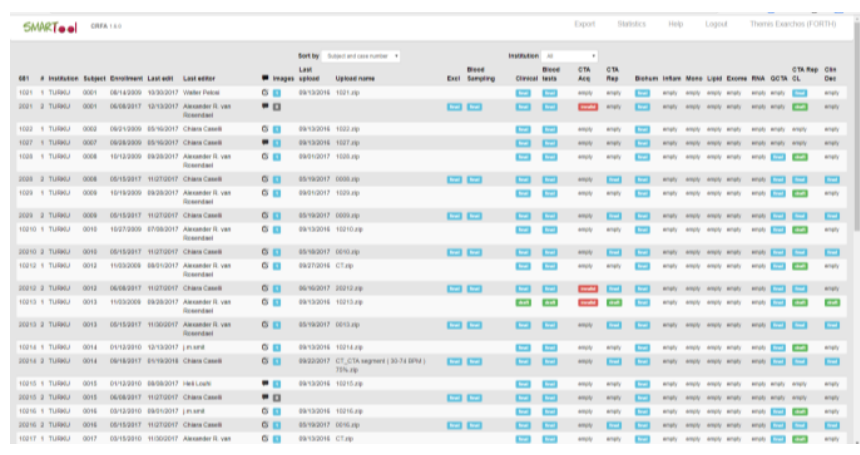

Figure 2. The CRFA platform of SMARTool patients' data collection and storage.

\section{CAD risk stratification}

A multimodal multiclass classification problem was defined for risk stratification based on the annotations provided by the medical experts of SMARTool and considering the stenosis degree. Thus, three classes are defined: (i) no CAD, (ii) obstructive CAD, (iii) nonobstructive CAD. The feature classes of demographics; clinical data; risk factors; symptoms; molecular variables (i.e. 
biohumoral, inflammatory markers and lipids profile); gene expression data; exposome; and monocytes were defined. The multimodal architecture consists of two processing layers which are defined according to late or intermediate data integration strategies. The first consists of: (i) decision tree-based prediction models (i.e. random forests, boosted decision trees) for each data view, whose individual decisions are effectively merged using simple mechanisms (e.g. weighted voting), or (ii) a multimodal deep neural network comprising appropriate deep learning subnetworks for each separate data view and, unifying their output into higher network layers. Intermediate data integration is based on multiple kernel learning. Kernel matrices are computed for each data view, and then they are combined, through a parametric linear function, in order to generate the final kernel matrix. Kernel-based classification (i.e. support vector machine, relevance vector machine) is subsequently applied to predict CAD risk stratification.

\section{CTCA imaging viewer}

A cloud-based repository is developed based on 3DnetMedical cloud solution and populated with retrospective and follow-up CCTA studies, which are retrieved from SMARTool CRFA. The viewer allows several visualization layouts and modes, including $2 \mathrm{D}$ and $3 \mathrm{D}$ manipulation, enabling annotation, comparing series and studies, creating snapshots and reports, and will integrate the functionalities to call the advanced modules for DSS (Fig. 3).

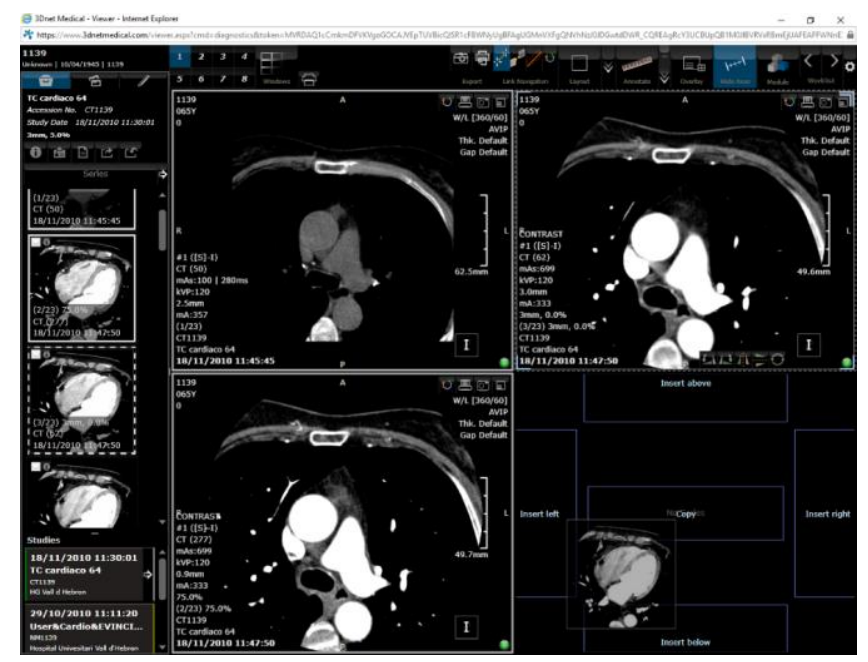

Figure 3. 3Dnet SMARTool Image viewer.

\section{E. $3 D$ reconstruction of arterial trees}

An innovative methodology and a software tool for 3D artery reconstruction of the entire coronary arterial tree based on CTCA images has been developed. The proposed methodology includes the following steps: pre-processing of CTCA images using a Frangi Vesselness filter [5], extraction of the vessel centerline using Multistencil Fast Marching Method (MSFM) [6], removal of blooming effect, estimation of membership distribution functions for the lumen, the outer wall and the calcified and non-calcified plaques (CP) intensities, implementation of an extension of active contour models using prior shapes for the lumen, the outer wall and the CP plaques segmentation based on Chan et al. [7] methodology and extraction of an adaptive intensity range for the detection of (NCP) in the plaque burden region. The major improvement of the segmentation algorithm is the incorporation of a prior shape comparison term into the energy function of the Level Set based model. In the final step, the segmented surfaces for each component are connected to construct the watertight $3 \mathrm{D}$ models. The proposed methodology has been implemented into an easy to use reconstruction tool.

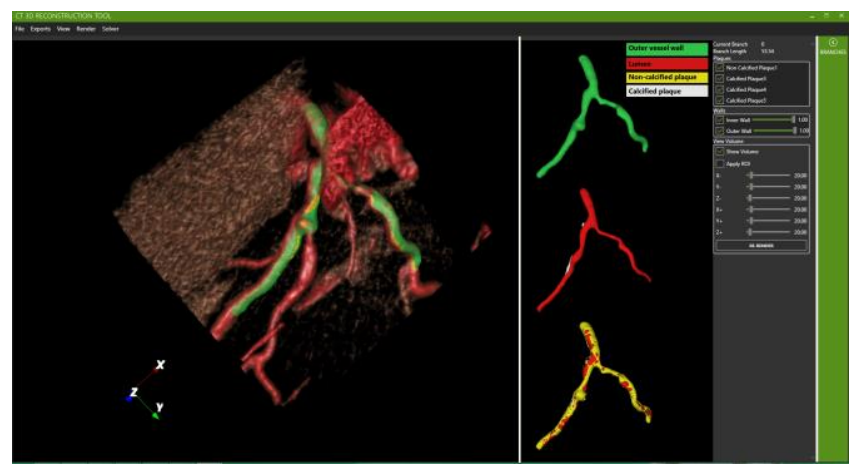

Figure 4. The 3D reconstruction tool for coronary reconstruction using CTCA images.

\section{F. Prognosis decision support system}

The prognosis DSS is based on the blood flow modelling, ESS estimation and plaque growth simulation. For this purpose the reconstructed geometries are utilized for finite element mesh discretization and an in house solver is used for the solution of the system of equations. In particular, NavierStokes are employed for blood flow, while convectiondiffusion equations are employed for modelling the mass transport in arterial lumen and wall [8,9]. Additionally, the oxidation of LDL, the inflammation caused by monocytes and macrophages and the formation of plaque due to foam cells formation and smooth muscle cells proliferation are modeled. The overall approach is presented in $[10,11]$.

\section{G. Diagnosis decision support system}

Diagnosis of the stenosis severity and the effect to hemodynamics is achieved invasively using a pressure wire for calculating FFR. An invasively measured FFR $<0.8$ is an indicator for percutaneous coronary intervention and stenting. In SMARTool a cost-effective approach is proposed which is employed in the reconstructed arteries using CTCA images providing in this way a non-invasive FFR calculation. We name the proposed index SmartFFR and its application is possible to reconstructed arteries with or without bifurcations. The approach is based on two blood flow calculations of 1 and $3 \mathrm{ml} / \mathrm{s}$ and the computation of the pressure gradient over the lesion, normalized by the ratio over the range of $0-4 \mathrm{ml} / \mathrm{s}$ for a normal artery. In case of bifurcation the Murray's law is implemented for the estimation of flow at each branch [2].

\section{H. Treatment decision support}

The DSS module for treatment is based on the computational modeling of stent deployment in the reconstructed arterial segments (Fig. 5). In addition, the comparison and evaluation of different procedural options taking into account not only the specific anatomical characteristics but also the mechanical properties of the 
arterial morphology, as well as the plaque type, could reveal useful clinical information and assist in the improvement of the interventional planning. In the proposed approach the stent geometry is placed in the stenosed arterial geometry and spatial grid generation is created for all the geometries (arterial wall, stent). The arterial wall is assumed to be a homogeneous hyperelastic material according to MooneyRivlin law and the stent a bilinear elasto-plastic material. A pressure boundary condition is imposed at the inner side of the stent, while the ends of the arterial segment are not allowed to move and rotate but they are free to move only in the axial and radial directions of the stent.

\section{RESULTS}

An initial dataset of 101 patients from SMARTool is used for the risk stratification algorithm. The implemented machine learning algorithms are feed-forward neural networks, support vector machine (SVM) and random forest classifiers. The SVM presents the highest accuracy when using the features of demographics, risk factors, symptoms, molecular systemic variables by applying a feature ranking technique according to the InfoGain criterion. Specifically, 25 and 76 patients are identified as no-CAD and mild to severe CAD, respectively. The accuracy, specificity and sensitivity are $85.1 \%, 98.7 \%$ and $44 \%$, respectively. The methodology of $3 \mathrm{D}$ reconstruction was compared to IVUS$\mathrm{VH}$ based reconstruction and the correlation coefficients for the degree of stenosis, the plaque burden, the minimal lumen area and the minimal lumen diameter, were $0.79,0.77,0.75$, $0.85,0.81$, respectively. SmartFFR has been validated compared to invasively measured FFR with a correlation coefficient of 0.90 proven to be an accurate diagnostic index for DSS. Plaque growth modelling demonstrates that the inclusion of variables such as the macrophages and foam cells concentrations can increase to $75 \%$ the prediction accuracy of regions prone to plaque formation providing a good accuracy for prognosis DSS. The stent expansion is affected by the degree of stenosis. It is observed that the stent does not follow a uniform expansion, while highest stresses are depicted near the connection of the stent struts. This information is useful for the clinical practice and the treatment DSS.

\section{DISCUSSION AND CONCLUSIONS}

SMARTool aims to develop a platform for risk stratification and DSS for diagnosis, prognosis and treatment. The preliminary results indicate that the developed algorithms and methodologies are accurate. More specifically, 3D reconstruction is validated using IVUS data and the accuracy is higher than the current literature. Additionally, the SmartFFR is an accurate diagnostic tool for PCI, while complex modelling of plaque growth could be used in the future for accurate prediction of regions which are prone to disease progression. The preliminary results of the effect of low ESS and high LDL concentration are in agreement with other studies [2, 3]. Finally, the risk stratification algorithm presents high accuracy and specificity, while the $44 \%$ of sensitivity means a reduction of unnecessary imaging and invasive interventions reducing the costs of CAD treatment and improving patient's life.

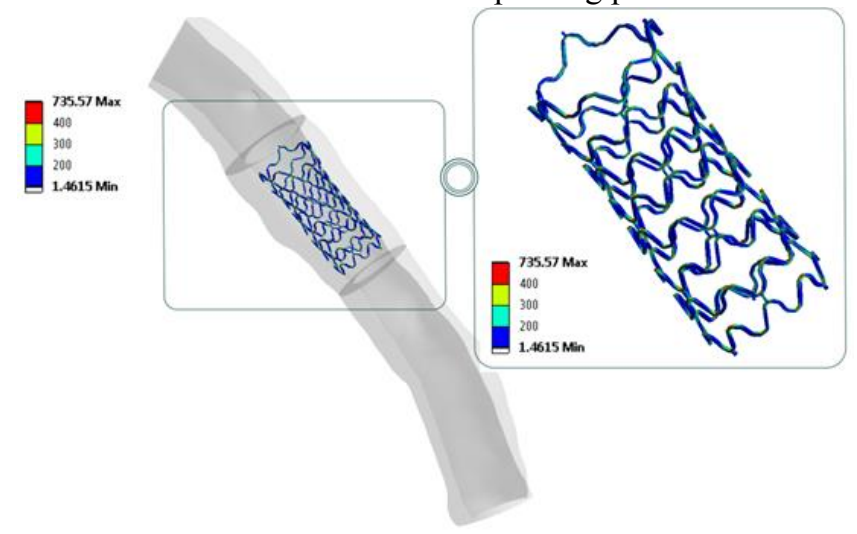

Figure 5. Von Mises stress (MPa) distribution for the stent in the unloading phase.

\section{REFERENCES}

[1] W. H. O. (WHO). The atlas of heart disease and stroke. Available: http://www.who.int/cardiovascular_diseases/en/cvd_atlas_16_death_f rom_stroke.pdf

[2] A. Sakellarios et al., "Prediction of atherosclerotic disease progression using LDL transport modelling: a serial computed tomographic coronary angiographic study," Eur Heart J Cardiovasc Imaging, Mar 162016.

[3] C. V. Bourantas et al., "Noninvasive Prediction of Atherosclerotic Progression: The PROSPECT-MSCT Study," JACC Cardiovasc Imaging, vol. 9, no. 8, pp. 1009-11, Aug 2016.

[4] R. Liga et al., "Multicentre multi-device hybrid imaging study of coronary artery disease: results from the EValuation of INtegrated Cardiac Imaging for the Detection and Characterization of Ischaemic Heart Disease (EVINCI) hybrid imaging population," Eur Heart J Cardiovasc Imaging, vol. 17, no. 9, pp. 951-60, Sep 2016.

[5] A. F. Frangi, W. J. Niessen, K. L. Vincken, and M. A. Viergever, "Multiscale vessel enhancement filtering," in Medical Image Computing and Computer-Assisted Intervention - MICCAI'98: First International Conference Cambridge, MA, USA, October 11-13, 1998 Proceedings, W. M. Wells, A. Colchester, and S. Delp, Eds. Berlin, Heidelberg: Springer Berlin Heidelberg, 1998, pp. 130-137.

[6] C. Metz, M. Schaap, A. Weustink, N. Mollet, T. van Walsum, and W. Niessen, "Coronary centerline extraction from CT coronary angiography images using a minimum cost path approach," Medical physics, vol. 36, no. 12, pp. 5568-5579, 2009.

[7] T. F. Chan and L. A. Vese, "Active contours without edges," IEEE Transactions on image processing, vol. 10, no. 2, pp. 266-277, 2001.

[8] A. I. Sakellarios et al., "Patient-specific computational modeling of subendothelial LDL accumulation in a stenosed right coronary artery: effect of hemodynamic and biological factors," (in English), American Journal of Physiology-Heart and Circulatory Physiology, vol. 304, no. 11, pp. H1455-H1470, Jun 2013.

[9] N. Sun, R. Torii, N. B. Wood, A. D. Hughes, S. A. Thom, and X. Y. $\mathrm{Xu}$, "Computational modeling of LDL and albumin transport in an in vivo CT image-based human right coronary artery," (in eng), $J$ Biomech Eng, vol. 131, no. 2, p. 021003, Feb 2009.

[10] A. Sakellarios et al., "Prediction of Atherosclerotic Plaque Development in an in Vivo Coronary Arterial Segment Based on a Multi-level Modeling Approach," IEEE Trans Biomed Eng, Oct 19 2016.

[11] O. Parodi et al., "Patient-Specific Prediction of Coronary Plaque Growth From CTA Angiography: A Multiscale Model for Plaque Formation and Progression," (in English), Ieee Transactions on Information Technology in Biomedicine, vol. 16, no. 5, pp. 952-965, Sep 2012. 